\title{
ON THE VALUE OF DETERMINANTS
}

\author{
JOHN H. E. COHN
}

The following problems were suggested, in the January 1962 issue of the Bulletin:

What are the maximum values of $n$th order determinants subject to the conditions

(a) each element, $a_{r s}=0$ or 1 ,

(b) each element, $a_{r s}=-1$ or 1 ,

(c) each element, $a_{r s}=-1,0$ or 1 ?

We shall not solve these problems completely, but we shall show that the three problems are equivalent and obtain the values approximately for large $n$.

Notation. Define $f(n), g(n), h(n)$ to be the maximum values of $n$th order determinants with elements subject to (a), (b), and (c) respectively and let $F_{n}, G_{n}, H_{n}$ be the matrices satisfying the conditions whose determinants have values $f(n), g(n)$ and $h(n)$. Of course these matrices are not unique.

\section{Preliminaries.}

THEOREM 1. $g(n)=h(n)$ for each $n$.

Certainly, since the class of matrices with elements $-1,0$ or 1 contains the class with elements -1 or 1 therefore, $h(n) \geqq g(n)$.

Secondly, consider $H_{n}$. If $H_{n}$ has no zero element then clearly $g(n)=h(n)$. If $H_{n}$ has at least one zero element, suppose $a_{r s}=0$. Then consider the expansion by the $r$ th row of $h(n) . h(n)=a_{r 1} A_{r 1}+a_{r 2} A_{r 2}$ $+\cdots+a_{r n} A_{r n}$. If $A_{r s}>0$, we could increase $h(n)$ by replacing $a_{r s}$ by 1 . If $A_{r s}<0$ we could increase $h(n)$ by replacing $a_{r s}$ by -1 . If $A_{r s}=0$ we could replace $a_{r s}$ by 1 without altering $h(n)$. Hence we may in turn replace each zero element of $H_{n}$ without decreasing $h(n)$.

Hence $g(n) \geqq h(n)$, and so

$$
g(n)=h(n) .
$$

Theorem 2. $g(n)=2^{n-1} f(n-1)$, for each $n$.

Consider $G_{n}=\left(a_{r s}\right)\left(a_{r s}= \pm 1\right)$. If $a_{1 s} \neq 1, a_{1 s}=-1$ and in this case, by multiplying each element in the sth column by -1 we obtain

$$
g(n)= \pm\left|\begin{array}{cc}
1 & 1 \cdots 1 \\
& b_{r s}
\end{array}\right| \quad b_{r s}= \pm 1, \quad r \geqq 2 .
$$

Received by the editors April 17, 1962. 
Similarly we can do the same for each element in the first column and obtain

$$
g(n)= \pm\left|\begin{array}{cc}
1 & 1 \cdots 1 \\
1 & \\
1 & \\
\vdots & c_{r s} \\
1 &
\end{array}\right| \quad c_{r s}= \pm 1, r, s \geqq 2
$$

Therefore, interchanging the second and third rows if the sign outside is minus we obtain

$$
g(n)=\left|\begin{array}{cc}
1 & 1 \cdots 1 \\
1 & \\
\vdots & d_{r s} \\
1 &
\end{array}\right| \quad d_{r s}= \pm 1, r, s \geqq 2
$$

Now subtract the first row from each of the others and we obtain

$$
g(n)=\left|\begin{array}{cc}
1 & 1 \cdots 1 \\
0 & \\
0 & d_{r s}-1 \\
\vdots & \\
0 &
\end{array}\right|
$$

But if $d_{r s}=-1$ or 1 , then $d_{r s}-1=0$ or -2 . Hence, expanding by the first column we obtain

$$
g(n)=(-2)^{n-1}\left|e_{r s}\right|_{(n-1)} \text { where } e_{r s}=0 \text { or } 1 .
$$

Hence $g(n) \leqq 2^{n-1} f(n-1)$.

Conversely,

$$
\begin{aligned}
& 2^{n-1} f(n-1)=2^{n-1}\left|a_{r s}\right|_{n-1} \quad a_{r s}=0 \text { or } 1 \\
& =\left|2 a_{r s}\right|_{n-1}
\end{aligned}
$$

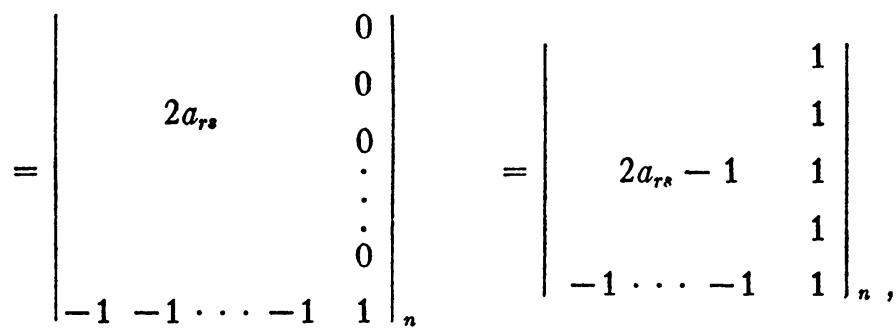


adding the last row to each of the others. But if $a_{r s}=0$ or 1 , then

$$
2 a_{r e}-1=1 \text { or }-1 \text { hence } 2^{n-1} f(n-1) \leqq g(n) \text {. }
$$

This concludes the proof, and shows incidentally that $g(n)$ is always a multiple of $2^{n-1}$.

This shows that the three problems are equivalent and so we shall concentrate on the second from now on.

We now prove the following results.

TheOREM 3. $g(n) \geqq(n-2) 2^{n-1}$.

For $g(n)$ is not less than the circulant

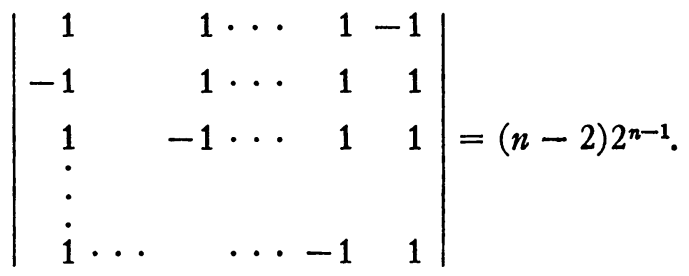

TheOREM 4. $g(n+1) \geqq 2 g(n)$.

For clearly $f(n) \geqq f(n-1)$ and so by Theorem 2 , the result follows.

THEOREM 5. $g(n) \leqq n^{n / 2}$.

This is an immediate corollary of Hadamard's inequality.

Theorem 6. $g(1)=1 ; g(2)=2$.

The first of these is trivial. For the second we observe that

$$
g(2) \geqq\left|\begin{array}{rr}
1 & 1 \\
-1 & 1
\end{array}\right|=2
$$

and $g(2) \leqq 2$, by Theorem 5 .

Theorem 7. For each $n, g(2 n) \geqq 2^{n}[g(n)]^{2}$.

Consider the $2 n \times 2 n$ matrix

$$
\left(\begin{array}{cc}
G_{n} & -G_{n} \\
G_{n} & G_{n}
\end{array}\right)
$$

In this each element is 1 or -1 .

Hence

$$
g(2 n) \geqq\left|\begin{array}{cc}
G_{n} & -G_{n} \\
G_{n} & G_{n}
\end{array}\right|=\left|\begin{array}{cc}
2 G_{n} & 0 \\
G_{n} & G_{n}
\end{array}\right|,=\left|2 G_{n}\right| \cdot\left|G_{n}\right|=2^{n}[g(n)]^{2} .
$$


TheOREM 8. If $n=2^{m}, g(n)=n^{n / 2}$.

By Theorem $5, g(n) \leqq n^{n / 2}$, and we prove by induction that $g(n)$ $\geqq n^{n / 2}$.

(a) We know that $g(2)=2$.

(b) Suppose that result is true for $n=2^{m_{0}}$.

Then, by Theorem 7

$$
\begin{aligned}
g(2 n) & \geqq 2^{n}[g(n)]^{2} \\
& \geqq 2^{n}\left[n^{n / 2}\right]^{2} \\
& =(2 n)^{n} .
\end{aligned}
$$

This concludes the proof.

TheOREM 9. $g(m n) \geqq[g(m)]^{n}[g(n)]^{m}$.

Consider $g(n)=\left|G_{n}\right|=\left|a_{r s}\right|, a_{r s}= \pm 1$. Then it is well-known that by adding and subtracting rows and columns we may reduce this determinant to the diagonal form

$$
g(n)=\left|\begin{array}{ccccc}
d_{1} & \cdot & \cdot & \cdot & \cdot \\
\cdot & d_{2} & & & \cdot \\
\cdot & & & & \cdot \\
\cdot & \cdot & \cdot & \cdot & d_{n}
\end{array}\right|=d_{1} d_{2} \cdots d_{n} .
$$

Now consider the $n m$ th order matrix

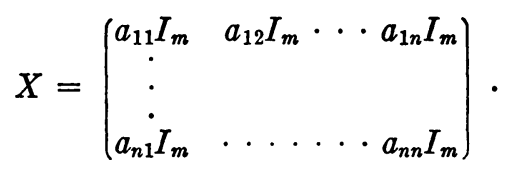

Now the same process of adding rows and columns which diagonalised $g(n)$ will ensure that

$$
\begin{aligned}
|X| & =\left|\begin{array}{cccc}
d_{1} I_{m} & 0 & \cdots & 0 \\
0 & d_{2} I_{m} & \cdot \\
\cdot & & \cdot \\
\cdot & & \cdot \\
0 & \cdots & \cdots & d_{n} I_{m}
\end{array}\right| \\
& =d_{1}^{m} d_{2}^{m} \cdots d_{n}^{m}=[g(n)]^{m} .
\end{aligned}
$$

Now consider 


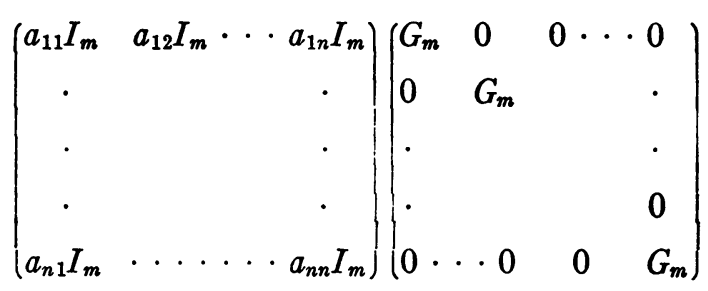

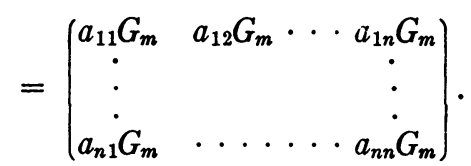

Now since each $a_{r s}= \pm 1$, all the elements in the matrix on the R.H.S. are \pm 1 . Hence

$$
g(n m) \geqq|X|\left|\begin{array}{cccc}
G_{m} & 0 & \cdots & 0 \\
0 & G_{m} & & \\
\vdots & & & \\
\cdot & & & G_{m}
\end{array}\right|=[g(n)]^{m}[g(m)]^{n} .
$$

This proves the theorem.

THEOREM 10. $g\left(m^{n}\right) \geqq[g(m)]^{n m^{n-1}}$.

For by Theorem 9

$\log g\left(m_{1} m_{2}\right) \geqq m_{1} \log g\left(m_{2}\right)+m_{2} \log g\left(m_{1}\right) 。$

Hence $\log g\left(m^{2}\right) \geqq 2 m \log g(m)$. Suppose

$$
\log g\left(m^{k}\right) \geqq k m^{k-1} \log g(m) .
$$

Then

$$
\begin{aligned}
\log g\left(m^{k+1}\right) & \geqq m k m^{k-1} \log g(m)+m^{k} \log g(m) \\
& =(k+1) m^{k} \log g(m) .
\end{aligned}
$$

Hence we have, by induction

$$
g\left(m^{n}\right) \geqq[g(m)]^{n m^{n-1}} .
$$

Theorem 11. $g(m) \leqq m g(m-1)$.

For,

$$
\begin{aligned}
g(m) & =\left|\begin{array}{rrr}
1 & 1 & \cdots \\
-1 & \text { or } & 1
\end{array}\right|_{m} \\
& =| \pm 1|_{m-1}-| \pm 1|_{m-1} \cdots(-)^{m-1}| \pm 1|_{m-1} \\
& \leqq m g(m-1) .
\end{aligned}
$$


As an immediate corollary we have, by Theorem 8:

Theorem 12. If $X=2^{m}, g(X-1) \geqq X^{x / 2-1}$.

Our central theorem which we shall prove is

Theorem 13. For all sufficiently large $n, g(n) \geqq n^{(1 / 2-\epsilon) n}$ for any given positive $\epsilon$.

In order to prove this, we shall require the following lemmas.

LEMMA I. For $x>2, \xi(x)$ is a monotonically increasing function, where

$$
\xi(x)=\frac{\log (x-1)}{\log \left(1+\frac{1}{x-1}\right)}
$$

and $\xi(x) \rightarrow \infty$ as $x \rightarrow \infty$.

This is fairly obvious.

LEMMA II. If $n \geqq(x-1)^{\xi(x)+1}$ then there exists an integer $\alpha$, such that $x^{\alpha} \geqq n \geqq(x-1)^{\alpha}$.

For, there certainly exists an integer $\alpha$ such that

$$
(x-1)^{\alpha+1} \geqq n \geqq(x-1)^{\alpha}
$$

and, moreover, $\alpha \geqq \xi(x)$ by the hypothesis. Hence

$$
\begin{aligned}
\frac{1}{\alpha} \leqq \frac{1}{\xi(x)} & =\frac{\log \left(\frac{x}{x-1}\right)}{\log (x-1)} \\
& =\frac{\log x-\log (x-1)}{\log (x-1)}
\end{aligned}
$$

therefore, $(1+\alpha) \log (x-1) \leqq \alpha \log x$ and so $(x-1)^{\alpha+1} \leqq x^{\alpha}$. Hence $x^{\alpha} \geqq(x-1)^{\alpha+1} \geqq n \geqq(x-1)^{\alpha}$.

LEMma III. If $\eta(x)=(a+x) /(b+c x)$ where $b, c$ are positive and $1 \leqq x \leqq d$ then $\eta(x)$ reaches its lowest value, either when $x=1$ or when $x=d$.

For $\eta^{\prime}(x)$ has constant sign and is continuous for $1 \leqq x \leqq d$.

LEMma IV. Given $n$, choose $x=X$ a power of 2 , satisfying Lemma II. Then there exist integers $\alpha, \beta$ such that $\alpha \geqq \xi(X)$ and $\alpha \geqq \beta \geqq 1$ and such that $X^{\beta}(X-1)^{\alpha-\beta} \geqq n \geqq X^{\beta-1}(X-1)^{\alpha-\beta+1}$. 
For by Lemma II, $\alpha \geqq \xi(X)$ and $X^{\alpha} \geqq n \geqq(X-1)^{\alpha}$. Hence

$$
\left(\frac{X}{X-1}\right)^{a} \geqq \frac{n}{(X-1)^{\alpha}} \geqq 1 \text {. }
$$

Hence there exists an integer $\beta$, such that $(X /(X-1))^{\beta} \geqq n /(X-1)^{\alpha}$ $\geqq(X /(X-1))^{\beta-1}$ and $\alpha \geqq \beta \geqq 1$. Hence $X^{\beta}(X-1)^{\alpha-\beta} \geqq n$ $\geqq(X-1)^{\alpha-\beta+1} X^{\beta-1}$.

We are now in a position to complete the proof. We have by Lemma IV,

$$
g(n) \geqq g\left[(X-1)^{\alpha-\beta+1} X^{\beta-1}\right] .
$$

Hence by Theorem 9

$$
\begin{aligned}
\log g(n) \geqq & (X-1)^{\alpha-\beta+1} \log g\left(X^{\beta-1}\right)+X^{\beta-1} \log g\left\{(X-1)^{\alpha-\beta+1}\right\} \\
\geqq & (X-1)^{\alpha-\beta+1}(\beta-1) X^{\beta-2} \log g(X) \\
& +X^{\beta-1}(\alpha-\beta+1)(X-1)^{\alpha-\beta} \log (X-1), \text { by Theorem 10, } \\
\geqq & (X-1)^{\alpha-\beta+1}(\beta-1) X^{\beta-2} \frac{1}{2} X \log X \\
& +X^{\beta-1}(\alpha-\beta+1)(X-1)^{\alpha-\beta}\left(\frac{1}{2} X-1\right) \log X
\end{aligned}
$$

by Theorems 8 and 12, since $X$ is a power of 2 . Hence $\log g(n)$

$$
\begin{gathered}
\geqq \frac{1}{2} \log X \cdot(X-1)^{\alpha-\beta} X^{\beta-1}[(\alpha-\beta+1)(X-2)+(\beta-1)(X-1)] \\
\quad \log g(n) \geqq \frac{1}{2} \log X \cdot(X-1)^{\alpha-\beta} X^{\beta-1}[\alpha(X-2)-1+\beta] .
\end{gathered}
$$

Also $X^{\beta}(X-1)^{\alpha-\beta} \geqq n$ and so

$$
n \log n \leqq X^{\beta}(X-1)^{\alpha-\beta}[(\alpha-\beta) \log (X-1)+\beta \log X] .
$$

Hence

$$
\frac{\log g(n)}{n \log n} \geqq \frac{\log X}{2 X} \frac{\alpha(X-2)-1+\beta}{\alpha \log (X-1)+\beta \log \left(\frac{X}{X-1}\right)}
$$

Now $\alpha \geqq \beta \geqq 1$ and so by Lemma III the lowest value of the expression on the right hand side occurs when either $\beta=1$ or $\beta=\alpha$. Hence

$$
\frac{\log g(n)}{n \log n} \geqq \min \{A, B\},
$$

where 


$$
\begin{aligned}
& A=\frac{\log X}{2 X} \frac{\alpha(X-2)}{\alpha \log (X-1)+\log \left(\frac{X}{X-1}\right)}, \\
& B=\frac{\log X}{2 X} \frac{\alpha(X-1)-1}{\alpha \log X}=\frac{\alpha(X-1)-1}{2 X \alpha} .
\end{aligned}
$$

Now $B=\frac{1}{2}-(1+\alpha) / 2 X \alpha$, but $\alpha \geqq \xi(X)>1$ hence $1+1 / \alpha<2$, and $B>\frac{1}{2}$ $-1 / X>\frac{1}{2}-\epsilon$ provided $X>1 / \epsilon$.

But, given $\epsilon>0$ we may choose a power $X$ of 2 such that $X>1 / \epsilon$. Then for every $n \geqq n_{0}=(X-1)^{\xi(X)+1}$ we have the above inequality. Also

$$
\begin{aligned}
A & =\frac{\log X}{2 X} \cdot \frac{X-2}{\log (X-1)} \cdot\left\{1-\frac{\log \frac{X}{X-1}}{\alpha \log (X-1)+\log \left(\frac{X}{X-1}\right)}\right\} \\
> & \frac{\log X}{2 X} \cdot \frac{X-2}{\log (X-1)}\left\{1-\frac{\log \frac{X}{X-1}}{\log (X-1)+\log \frac{X}{X-1}}\right\} \\
& =\frac{\log X}{2 X} \cdot \frac{X-2}{\log (X-1)}\left\{\frac{\log (X-1)}{\log X}\right\} .
\end{aligned}
$$

Hence $A>\frac{1}{2}-1 / X>\frac{1}{2}-\epsilon$, and for all sufficiently large $n$,

$$
\frac{\log g(n)}{n \log n}>\frac{1}{2}-\epsilon
$$

i.e., $g(n)>n^{n(1 / 2-\epsilon)}$ which concludes the proof of Theorem 13.

Bedford College, London, England 